QUARTERLY OF APPLIED MATHEMATICS

VOLUME LXX, NUMBER 1

MARCH 2012, PAGES 53-67

S $0033-569 X(2011) 01230-8$

Article electronically published on September 16, 2011

\title{
MODELING THE FLASH-HEAT EXPERIMENT ON POROUS DOMAINS
}

\author{
$\mathrm{BY}$ \\ H. T. BANKS (Department of Mathematics, Center for Research in Scientific Computation, North \\ Carolina State University, Raleigh, North Carolina 27695-8212), \\ D. CIORANESCU (Laboratoire J. L. Lions, Université Pierre et Marie Curie, 175 rue du \\ Chevaleret, 75013 Paris, France), \\ A. K. CRINER (Department of Mathematics, Center for Research in Scientific Computation, North \\ Carolina State University, Raleigh, North Carolina 27695-8212), \\ AND \\ W. P. WINFREE (Nondestructive Evaluation Science Branch, NASA Langley Research Center, MS
} 231, Hampton, Virginia 23681)

\begin{abstract}
We discuss a mathematical model for the flash-heat experiment in homogeneous isotropic media. We then use this model to investigate the use of homogenization techniques in approximating models for interrogation via flash-heating in porous materials. We represent porous materials as both randomly perforated domains and periodically perforated domains.
\end{abstract}

1. Introduction. Nondestructive evaluation (NDE) is an important tool in many fields. Nondestructive evaluation is used to identify damage in structures, including components of aircraft, spacecraft, automobiles, trains and piping, as they age beyond their design life. Proper use of nondestructive evaluation can increase the safety and service life of components of many structures. Ultrasound, magnetic particle imaging, eddy current, acoustic emission and radiology are a few examples of NDE techniques. These techniques have been developed in a large number of applications, particularly for homogeneous metallic materials [3]-[7]. Composite materials are increasingly popular in structures including aeronautical and aerospace vehicles and are constructed with an acceptable level of porosity. This porosity causes a certain amount of noise in the interrogation signal of any NDE technique. In [1], where we investigated using active thermography to detect damage in porous domains, we found that we could detect damage of a certain size within a porous medium using thermal interrogation. However, the methods developed in [1] were too computationally intensive to use in the sophisticated

Received May 16, 2010.

2000 Mathematics Subject Classification. Primary 35B27, 76R50, 78M40.

Key words and phrases. Modeling porous media, thermal diffusion, homogenization. 
parameter estimation routines needed to characterize damage. As in [1] we treat here the problem of modeling the flow of heat in a porous domain but in the current work we focus on the techniques of homogenization theory (which are less computationally intensive than the methods used in [1]) to capture the behavior.

We aim to use homogenization theory to model a two-dimensional cross section version of a flash-heat experiment in porous domains. In [2, we presented four different scenarios: (i) heat in a perfectly insulated two-dimensional sample (system (2) below); (ii) heat flow in a cross section with part of the boundary held at a fixed temperature; (iii) heat flow in a cross section with loss only in the direction orthogonal to the cross section with boundaries otherwise insulated; and (iv) heat flow in a cross section with small loss on either part or all of its boundary. Our current efforts focus on (iv) with loss on all of the boundary of the cross section (system (3) below). In Section 2 , we discuss the mathematical model corresponding to the physical assumptions of (iv) with uniform heat loss on the boundaries with anisotropic flow. We then discuss the finite element method, which is used to solve all of the partial differential equations in our current efforts, in Section 3 We also discuss the procedures used in homogenization to approximate the flow of heat over a domain with random pores by the flow of heat over a domain with many periodically placed pores. One passes this periodic domain to a limit using homogenization theory [8]-[1] to gain a limit partial differential equation which replaces the periodically perforated domain with a homogeneous domain that has anisotropic flow approximating the effect of the porosity. With the results of homogenization theory developed in Section 4 we then summarize and compare the results of simulations of the flash-heat experiment on a randomly perforated domain, a periodically perforated domain, and a homogeneous domain with the anisotropic flow derived from homogenization theory in Section 5. We also graphically analyze example simulations in Section [5]

2. Mathematical model. We first recall the system (1) which corresponds to the physical flash-heat experiment described in [1]. This experiment assumes that the temperature of the specimen is within the solid state phase and the boundaries are perfectly insulated [13. We model the flash-heat experiment on an $L_{1}$ (length in the $x$ direction) by $L_{2}$ (length in the $y$ direction) rectangle during the time interval $t \in(0, T)$ with $T<\infty$. We refer to the $L_{1}$ by $L_{2}$ rectangle as $\widehat{\Omega}$ and the four boundaries $\omega_{i}$, for $i \in\{1,2,3,4\}$. When referring to the entire boundary of $\widehat{\Omega}$, we use $\partial \widehat{\Omega}=\bigcup_{i=1}^{4} \omega_{i}$. We take, $L_{1}=2 \mathrm{~mm}$ and $L_{2}=1 \mathrm{~mm}$. The bottom boundary, $\omega_{4}=\left\{(x, y) \mid y=0, x \in\left(0, L_{1}\right)\right\}$, is heated with heat flux $S_{0}=3.3 \times 10^{-3} \frac{\mathrm{W}}{\mathrm{mm}^{2}}$ from the initial time, $t_{0}=0$, until $t_{s}$ and insulated for $t>t_{s}$. We use an indicator function to describe the flash-heating of the boundary,

$$
\mathcal{I}_{\left[t_{0}, t_{s}\right]}(t)= \begin{cases}1 & \text { for } t \in\left[t_{0}, t_{s}\right] \\ 0 & \text { otherwise }\end{cases}
$$

The other boundaries are insulated throughout the experiment. The boundary locations are depicted in Figure 1. This experiment can be described by the system 


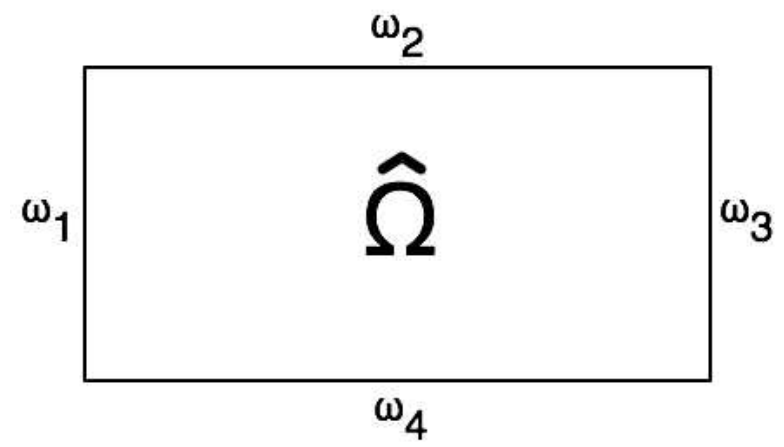

FIG. 1. The homogeneous rectangle with boundaries.

$$
\begin{cases}c_{p} \rho \hat{u}_{t}-k \Delta \hat{u}=0 & \text { in } \widehat{\Omega} \times(0, T) \\ k \frac{\partial \hat{u}}{\partial \eta}=S_{0} \mathcal{I}_{\left[t_{0}, t_{s}\right]}(t) & \text { on } \omega_{4} \times(0, T) \\ k \frac{\partial \hat{u}}{\partial \eta}=0 & \text { on } \bigcup_{i=1}^{3} \omega_{i} \times(0, T) \\ \hat{u}(0, \vec{x})=u_{0} & \text { for all } \vec{x} \in \widehat{\Omega},\end{cases}
$$

with thermal conductivity $k=3.5 \times 10^{-3} \mathrm{~J} /(\mathrm{mm} \mathrm{K} \mathrm{sec})$, specific heat $c_{p}=0.75 \mathrm{~J} /(\mathrm{g} \mathrm{K})$, material density $\rho=1.6 \times 10^{-3} \mathrm{~g} /\left(\mathrm{mm}^{3}\right)$, and the dependent variable $\hat{u}$ is temperature in degrees Kelvin. The values used throughout this article are summarized in Table 1. We will now make a few changes to the above system. In the partial differential equation, $\Delta u$ may be written more generally as $\nabla \cdot\left(A^{0} \nabla u\right)$, where $A^{0}$ is the $2 \times 2$ identity matrix $I_{2}$ in the present section and Section 2. We make this change because subsequently we will replace the identity with another positive definite matrix, which will be derived from homogenization theory. Corresponding to this change, we must also change the boundary conditions, so that the boundary conditions still specify the flux when $A^{0}$ is not the identity matrix. Specifically, $\frac{\partial u}{\partial \eta}=n \cdot \nabla u$, where $n$ is the exterior unit normal vector, is replaced with $\frac{\partial u}{\partial \eta_{A^{0}}}=n \cdot A^{0} \nabla u$. For convenience and without loss of generality, we will also translate the temperature so that the initial temperature, which we will take to also be the surrounding temperature, is zero. This corresponds to the change $u=\hat{u}-u_{0}$. We wish to use the thermal diffusivity, $\alpha=\frac{k}{c_{p} \rho}$, as a characteristic parameter, so our final system is

$$
\begin{cases}u_{t}-\alpha \nabla \cdot\left(A^{0} \nabla u\right)=0 & \text { in } \widehat{\Omega} \times(0, T) \\ \alpha \frac{\partial u}{\partial \eta_{A^{0}}}=S_{f} \mathcal{I}_{\left[t_{0}, t_{s}\right]}(t) & \text { on } \omega_{4} \times(0, T) \\ \alpha \frac{\partial u}{\partial \eta_{A^{0}}}=0 & \text { on } \bigcup_{i=1}^{3} \omega_{i} \times(0, T) \\ u(\vec{x}, 0)=0 & \text { for all } \vec{x} \in \widehat{\Omega},\end{cases}
$$

where $S_{f}=\frac{S_{0}}{c_{p} \rho}$.

We are using these results to model experiments which are being carried out at NASA Langley Research Center. The zero flux boundary conditions of (2) correspond to the physical assumption of a perfectly insulated boundary, which may not be reasonable in 
TABLE 1. Parameter values used throughout this document, unless otherwise mentioned. The $2 \times 2$ matrix, $A^{0}$, is a positive definite matrix arising from homogenization theory.

\begin{tabular}{||l|l|l||}
\hline Parameter & Value & Units \\
\hline$\left[t_{0}, t_{s}\right]$ & {$[0,0.6]$} & $\mathrm{s}$ \\
\hline$T$ & 5 & $\mathrm{~s}$ \\
\hline$S_{f}$ & 2.75 & $\mathrm{~K} \mathrm{~mm} / \mathrm{s}$ \\
\hline$\alpha$ & 2.9167 & $\mathrm{~mm}^{2} / \mathrm{s}$ \\
\hline$L_{1}$ & 2 & $\mathrm{~mm}$ \\
\hline$L_{2}$ & 1 & $\mathrm{~mm}$ \\
\hline & {$\left[\begin{array}{ll}1 & 0 \\
0 & 1\end{array}\right]$} & \\
$I_{2}$ &
\end{tabular}

some cases. We relax this assumption by replacing the boundary condition with Robin boundary conditions that correspond to the assumption that Newton cooling occurs on the boundaries. This is succinctly incorporated into system (3) below, which includes a $-\lambda u$ term in all of the boundary conditions:

$$
\begin{cases}u_{t}-\alpha \nabla \cdot\left(A^{0} \nabla u\right)=0 & \text { in } \widehat{\Omega} \times(0, T) \\ \alpha \frac{\partial u}{\partial \eta_{A^{0}}}=S_{f} \mathcal{I}_{\left[t_{0}, t_{s}\right]}(t)-\lambda u & \text { on } \omega_{4} \times(0, T) \\ \alpha \frac{\partial u}{\partial \eta_{A^{0}}}=-\lambda u & \text { on } \bigcup_{i=1}^{3} \omega_{i} \times(0, T) \\ u(\vec{x}, 0)=0 . & \end{cases}
$$

It is important to recall that $u=\hat{u}-u_{0}$, where $\hat{u}$ is the temperature and $u_{0}$ is both the initial temperature and the temperature surrounding the specimen. System (3) is a generalization of (2) in that (3) is identical to (2) for $\lambda=0$.

3. Numerical solutions. We will use the finite element method to numerically solve (3). The finite element method approximates the infinite-dimensional solution of a partial differential equation with a finite-dimensional approximation. The domain $(\widehat{\Omega})$ is discretized using the Delaunay triangulation. The finite-dimensional solution is taken from the space of piecewise two-dimensional affine functions, where the solution is affine on each mesh element (see [12] and [14] for details). Recall the notation $n=\left(n_{x}, n_{y}\right)$, where $n$ is the unit outward normal and $\frac{\partial u}{\partial \eta_{A^{0}}}=n \cdot\left(A^{0} \nabla u\right)$. The boundary conditions 
can also be written as

$$
\begin{array}{lll}
\alpha \frac{\partial u}{\partial \eta_{A^{0}}}=\alpha\left(a_{11} \frac{\partial u}{\partial x}+a_{12} \frac{\partial u}{\partial y}\right)=-\lambda u & \text { on } \omega_{3}, \\
\alpha \frac{\partial u}{\partial \eta_{A^{0}}}=\alpha\left(-a_{11} \frac{\partial u}{\partial x}-a_{12} \frac{\partial u}{\partial y}\right)=-\lambda u & \text { on } \omega_{1}, \\
\alpha \frac{\partial u}{\partial \eta_{A^{0}}}=\alpha\left(a_{21} \frac{\partial u}{\partial x}+a_{22} \frac{\partial u}{\partial y}\right)=-\lambda u & \text { on } \omega_{2}, \\
\alpha \frac{\partial u}{\partial \eta_{A^{0}}}=\partial \alpha\left(-a_{21} \frac{\partial u}{\partial x}-a_{22} \frac{\partial u}{\partial y}\right)=S_{f} \mathcal{I}_{\left[t_{0}, t_{s}\right]}(t)-\lambda u & \text { on } \omega_{4} .
\end{array}
$$

Using Green's identities, we obtain the weak form

$$
\begin{aligned}
\int_{\widehat{\Omega}} \phi(\vec{x}) u_{t} d \vec{x}+\alpha \int_{\widehat{\Omega}} \phi(\vec{x}) \nabla \phi \cdot A^{0} \nabla u d \vec{x} & -\int_{\omega_{4}} \phi(x, 0) S_{f} \mathcal{I}_{\left[t_{0}, t_{s}\right]}(t) d x \\
& +\lambda \int_{\partial \widehat{\Omega}} \phi u d s=0
\end{aligned}
$$

or

$$
\left\langle u_{t}, \phi\right\rangle+\alpha\left\langle\nabla \phi, A^{0} u\right\rangle-\int_{\omega_{4}} \phi(x, 0) S_{f} \mathcal{I}_{\left[t_{0}, t_{s}\right]}(t) d x+\lambda \int_{\partial \widehat{\Omega}} \phi u d s=0,
$$

where $\langle\phi, v\rangle=\int_{\widehat{\Omega}} \phi v d \vec{x}$. Here $\phi$ is a member of the space of test functions $H^{1}(\widehat{\Omega})$. We approximate $u$ with $u_{N}$ by

$$
u_{N}(t, \vec{x})=\sum_{i=1}^{N} T_{i}(t) \phi_{i}(\vec{x}) .
$$

Substituting this expression into the weak form with test functions $\phi_{i}, i \in\{1,2, \ldots, N\}$ in the space of two-dimensional affine functions (we refer the reader to [14 for more details on these basis elements and the time-dependent coefficients $T_{i}(t)$ ), we have

$$
\begin{aligned}
& \left\langle\frac{\partial}{\partial t}\left(\sum_{i=1}^{N} T_{i}(t) \phi_{i}\right), \phi_{j}\right\rangle+\alpha\left\langle\nabla \phi_{j}, A^{0} \nabla\left(\sum_{i=1}^{N} T_{i}(t) \phi_{i}\right)\right\rangle \\
& -\int_{\omega_{4}} \phi_{j}(x, 0) S_{f} \mathcal{I}_{\left[t_{0}, t_{s}\right]}(t) d x+\lambda \int_{\partial \widehat{\Omega}} \sum_{i=1}^{N} T_{i}(t) \phi_{i} \phi_{j} d s=0 .
\end{aligned}
$$

We may factor the time-dependent coefficients $T_{i}(t)$ of the basis elements $\phi_{i}(\vec{x})$ from the inner product to obtain

$$
\begin{aligned}
& \sum_{i=1}^{N} \frac{d}{d t} T_{i}\left\langle\phi_{i}, \phi_{j}\right\rangle+\alpha \sum_{i=1}^{N} T_{i}\left\langle\nabla \phi_{j}, A^{0} \nabla \phi_{i}\right\rangle \\
& -S_{f} \mathcal{I}_{\left[t_{0}, t_{s}\right]}(t) \int_{\omega_{4}} \phi_{j}(x, 0) d x+\sum_{i=1}^{N} T_{i} \int_{\partial \widehat{\Omega}} \phi_{i} \phi_{j} d s=0 .
\end{aligned}
$$


Equation (6) must be true for arbitrary $j \in\{1,2, \ldots, N\}$ and the system may be written as

$$
C \frac{d}{d t} \vec{T}(t)+(\alpha M+\lambda D) \vec{T}(t)=S_{f} \mathcal{I}_{\left[t_{0}, t_{s}\right]}(t) \vec{f}
$$

where $C$ is an $N \times N$ positive definite matrix with elements $c_{i j}=\left\langle\phi_{i}, \phi_{j}\right\rangle, M$ is an $N \times N$ positive definite matrix with elements $m_{i j}=\left\langle\nabla \phi_{i}, A^{0} \nabla \phi_{j}\right\rangle, D$ is an $N \times N$ matrix with components $d_{i j}=\int_{\partial \widehat{\Omega}} \phi_{i} \phi_{j} d s, \vec{f}$ is an $N$-vector with components $f_{i}=\int_{\omega_{4}} \phi_{i}(x, 0) d x$ and $\vec{T}$ is an $N$-component column vector. We verified our calculations of these arrays in the case where $A^{0}=I_{2}$ by comparing them to the corresponding values used to calculate the finite element method solution in MatLab's PDE toolbox [12].

4. Homogenization. Now that we have considered a model of the flash-heat experiment on a homogeneous domain, we will model the flash-heat experiment on more complicated domains than the rectangle $\widehat{\Omega}$. We developed a method to model the flash-heat experiment on porous domains in [1]. Though these simulations were useful in detecting damage, they were too computationally intensive for the more sophisticated parameter estimation procedures needed to characterize damage. Here we will use (33) along with random geometries to model the flash-heat experiment in a porous domain and compare this model to a limit partial differential equation which is derived from homogenization theory. In this formulation $A^{0}$ is no longer $I_{2}$ and the random complicated geometry is replaced with a less complicated domain with anisotropic flow. When comparing the subsequent models, we will focus on values of solutions (i.e., observations) obtained on the source boundary $\omega_{4}$ (a boundary on which we imagine sensors for observations in an inverse problem formulation for interior damage detection).

We will consider a random geometry $\Omega$ (depicted in Figure 2), which is composed of $\widehat{\Omega} \backslash \bigcup_{i=1}^{n_{r}} \Omega_{i}$, where $\Omega_{i}$ are $n_{r}$ randomly placed pores, $\partial \Omega_{i}$ is the boundary of the $i$ th pore and $\omega_{i}$ are the same as in the previous sections. We must now pose our problem on $\Omega$. We will call the dependent variable of this system $u^{\text {rand }}$, where 'rand' refers to the random domain. Here it is worthwhile to note that we have performed the transformations detailed in the beginning of Section 2 to all of the partial differential equations in this section.

The system corresponding to (3) on the random domain $\Omega$ is given by

$$
\begin{cases}u_{t}^{\text {rand }}-\alpha \Delta u^{\text {rand }}=0 & \text { on } \Omega \times(0, T) \\ \alpha \frac{\partial}{\partial \eta} u^{\text {rand }}=0 & \text { on } \bigcup_{i=1}^{n_{r}} \partial \Omega_{i} \times(0, T) \\ \alpha \frac{\partial}{\partial \eta} u^{\text {rand }}=-\lambda u^{\text {rand }} & \text { on } \bigcup_{i=1}^{3} \omega_{i} \times(0, T) \\ \alpha \frac{\partial}{\partial \eta} u^{\text {rand }}=S_{f} \mathcal{I}_{\left[0, t_{s}\right]}(t)-\lambda u^{\text {rand }} & \text { on } \omega_{4} \times(0, T) \\ u^{\text {rand }}(\vec{x}, 0)=0 . & \end{cases}
$$

We will use (8) to simulate data that one might expect from the flash-heat experiment performed on porous specimens. We generate these geometries and solve the partial differential equations using the methods developed in 1 .

In order to apply homogenization theory to (8), we will use geometries with sufficiently many pores to suppose that $\Omega$ has a periodic structure (though it may be that the physical 


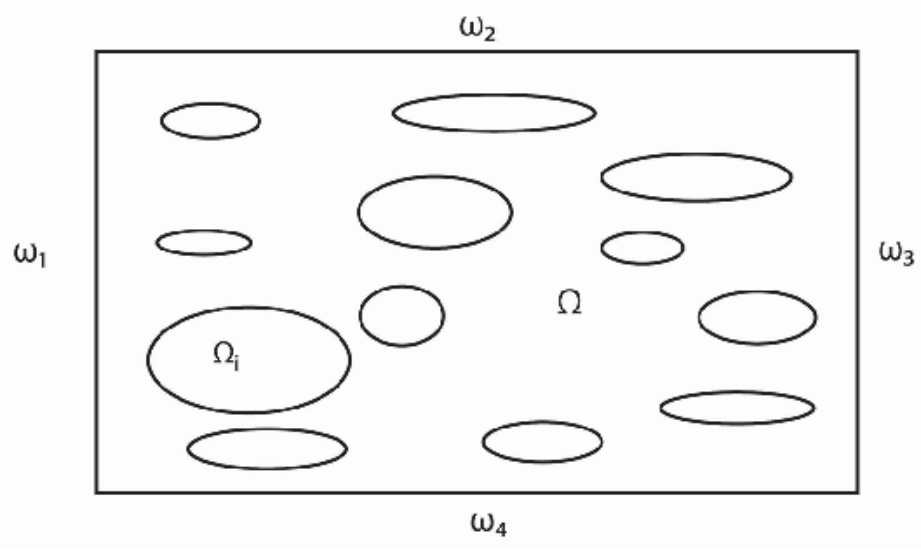

FIG. 2. A typical porous domain with random pores (enlarged view)

specimens are better modeled with periodic pores as the pores in composite materials are often the result of sinusoidal manufacturing processes). In other words, we suppose that the pores (or holes) are periodically distributed with a period $\varepsilon$, where $\varepsilon$ is a small parameter that we pass to zero in the limit. This is the framework of the periodic homogenization theory, which is explained in more detail in [10. To do so, we introduce a reference cell (or domain) $Y$. For our purpose, we take as $Y$ the original (homogeneous) rectangle $\widehat{\Omega}$. Let $B=\bigcup_{i=1}^{N} B_{i}$ be a set of $N$ open subsets strictly included in $Y$ such that $B_{i} \cap B_{j}=\emptyset$ for $i \neq j$.

Denote by $\tau(\varepsilon \bar{B})$ the set of all translated images of $\varepsilon \bar{B}$ of the form $\varepsilon(\kappa \ell+B), \kappa \in \mathbb{Z}^{2}$, $\kappa \ell=\left(\kappa_{1} \ell_{1}, \kappa_{2} \ell_{2}\right)$, so the set $\tau(\varepsilon \bar{B})$ represents the periodic pores in $\mathbb{R}^{2}$. Let $B^{\varepsilon}$ be the set of the holes contained in $\widehat{\Omega}$. With the above choice of $Y$, and taking for instance $\varepsilon=\frac{1}{\left[\sqrt{\frac{n_{\varepsilon}}{N}}\right]}$ with $n_{\varepsilon} \rightarrow+\infty$, no hole from $B^{\varepsilon}$ will intersect the boundary $\partial \widehat{\Omega}$. Here $\lceil\cdot\rfloor$ is the nearest integer function. We will set

$$
\Omega_{\varepsilon}=\widehat{\Omega} \backslash \bar{B}^{\varepsilon} .
$$

By this construction, the physical domain $\Omega_{\varepsilon}$ is periodically perforated with holes of size of the same order as the period. We are essentially approximating the random geometry $\Omega$ with the periodic geometry $\Omega_{\varepsilon}$.

We will use the following notation:

- $Y^{*}=Y \backslash B$,

- $X^{\dagger}$, the topological dual space of $X$,

- $\theta=\frac{\left|Y^{*}\right|}{|Y|}$, the proportion of the material in the cell $Y$,

- $|\omega|=$ the Lebesgue measure of any open set $\omega$,

- $\mathcal{M}_{\omega}(\varphi)=\frac{1}{|\omega|} \int_{\omega} \varphi(x) d x$, the mean value of $\varphi$ on the set $\omega$.

Observe that by construction, $\theta$ is also the proportion of the material in $\Omega_{\varepsilon}$ for any $\varepsilon>0$ and the percent porosity $=(1-\theta) \times 100 \%$. We are now prepared to consider our 
system on $\Omega_{\varepsilon}$. Using $\Omega_{\varepsilon}$ to approximate $\Omega$ in ( 8 ), we have

$$
\begin{cases}u_{t}^{\varepsilon}-\alpha \Delta u^{\varepsilon}=0 & \text { in } \Omega_{\varepsilon} \times(0, T) \\ \alpha \frac{\partial}{\partial \eta} u^{\varepsilon}=0 & \text { on } \partial B^{\varepsilon} \times(0, T) \\ \alpha \frac{\partial}{\partial \eta} u^{\varepsilon}=-\lambda u^{\varepsilon} & \text { on } \bigcup_{i=1}^{3} \omega_{i} \times(0, T) \\ \alpha \frac{\partial}{\partial \eta} u^{\varepsilon}=S_{f} \mathcal{I}_{\left[0, t_{s}\right]}(t)-\lambda u^{\varepsilon} & \text { on } \omega_{4} \times(0, T) \\ u^{\varepsilon}(\vec{x}, 0)=0 . & \end{cases}
$$

The system (91) possesses a unique solution in the Banach space

$$
\mathcal{W}_{\varepsilon}=\left\{v \mid v \in L^{2}\left(0, T ; H^{1}\left(\Omega_{\varepsilon}\right)\right), \frac{\partial v}{\partial t} \in L^{2}\left(0, T ;\left(H^{1}\left(\Omega_{\varepsilon}\right)\right)^{\dagger}\right)\right\} .
$$

One is then allowed to pass to the limit in (9) to obtain a limit homogenized system (for details, we refer the reader to [9, 10, 11]). In particular, these references contain proofs that

$$
\widetilde{u}^{\varepsilon} \rightarrow \theta U \quad \text { weakly in } L^{2}\left(0, T ; H^{1}(\widehat{\Omega})\right),
$$

where $U$ is the unique solution of the homogenized problem and $\widetilde{u}^{\varepsilon}$ is the zero extension of $u^{\varepsilon}$ from $\Omega_{\varepsilon}$ to the entire domain $\widehat{\Omega}$.

The limit system corresponding to (9) is given by

$$
\begin{cases}\theta U_{t}-\alpha \nabla \cdot\left(A^{0} U\right)=0 & \text { in } \widehat{\Omega} \times(0, T) \\ \alpha \frac{\partial U}{\partial \eta_{A^{0}}}=-\lambda U & \text { on } \bigcup_{i=1}^{3} \omega_{i} \times(0, T) \\ \alpha \frac{\partial U}{\partial \eta_{A^{0}}}=S_{f} \mathcal{I}_{\left[0, t_{s}\right]}(t)-\lambda U & \text { on } \omega_{4} \times(0, T) \\ U(\vec{x}, 0)=0 & \end{cases}
$$

The homogenized operator $A^{0}$ has constant coefficients and is expressed in terms of the following cell problems defining the "corrector" functions $\chi_{1}$ and $\chi_{2}$ :

$$
\left\{\begin{array}{l}
-\Delta \chi_{j}=0 \text { for } j=1,2 \text { in } Y^{*} \\
\chi_{j} \text { is } Y \text { periodic } \\
\frac{\partial}{\partial \eta}\left(\chi_{j}-y_{j}\right)=0 \text { on } \partial \Omega_{i} \\
\mathcal{M}_{Y}^{*}(\chi)=0 .
\end{array}\right.
$$

The homogenized matrix $A^{0}=\left(a_{i j}^{0}\right)$ is then defined by

$$
\begin{array}{ll}
a_{11}^{0}=\theta-\frac{1}{|Y|} \int_{Y^{*}} \frac{\partial \chi_{1}}{\partial y_{1}} d y, \quad a_{12}^{0}=-\frac{1}{|Y|} \int_{Y^{*}} \frac{\partial \chi_{2}}{\partial y_{1}} d y, \\
a_{21}^{0}=-\frac{1}{|Y|} \int_{Y^{*}} \frac{\partial \chi_{1}}{\partial y_{2}} d y, \quad a_{22}^{0}=\theta-\frac{1}{|Y|} \int_{Y^{*}} \frac{\partial \chi_{2}}{\partial y_{2}} d y .
\end{array}
$$

It is important to note that $\partial \Omega_{i}$ in (12) refers to the boundaries of the pores of the reference cells, depicted in Figure 3 .

It can be shown that the error estimate (distance between $\widetilde{u}^{\varepsilon}$ and $\theta U$ in the $L^{2}\left(0, T ; H^{1}(\widehat{\Omega})\right)$-norm) is of order of $\sqrt{\varepsilon}$, which completely justifies the homogenization procedure if $\varepsilon$ is sufficiently small, or equivalently if the number $n_{\varepsilon}$ of holes is sufficiently 


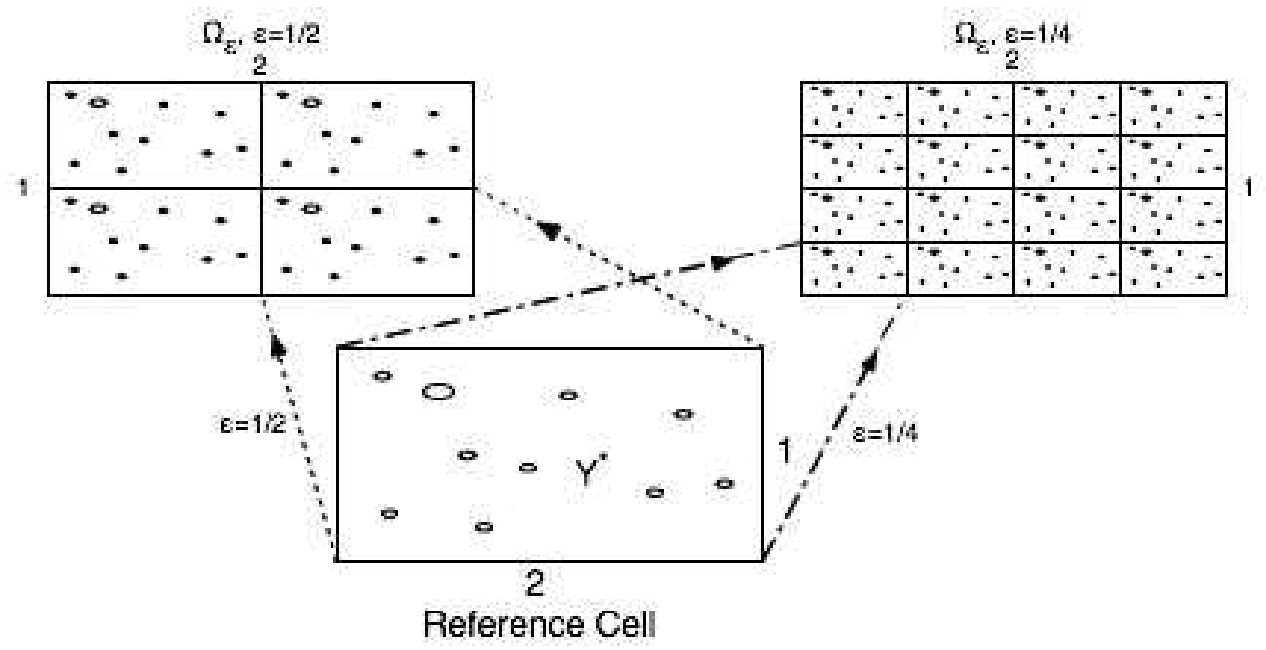

FIG. 3. The reference domain and the limit domain with $\varepsilon=\frac{1}{2}$ and $\varepsilon=\frac{1}{4}$.

large since $n_{\varepsilon} \sim \frac{1}{\varepsilon^{2}}$. Using the linear trace operator, $\gamma: H^{1}(\widehat{\Omega}) \rightarrow L^{2}\left(\omega_{4}\right)$ and arguments similar to those in [8], we obtain the result that $\gamma\left(\widetilde{u}^{\varepsilon}\right)$ converges weakly in $L^{2}\left(\omega_{4}\right)$ to $\gamma(U)$.

Recalling the convergence of $\widetilde{u}^{\varepsilon}$ to $U$, we created numerical simulations to compare $U$ to $u^{\varepsilon}$ and $u^{\text {rand }}$ on $\omega_{4}$. We used methods developed in [1] and [15] to generate the random geometries $\Omega$ and $Y^{*}$. We used MatLab's PDE toolbox, as in 1], to then solve the partial differential equations on these domains. To calculate $U$, we used the finite element schemes detailed in Section 2

For each simulation, let $N$ be the number of pores in the reference cell, and $n_{r}$ the number of pores in the random geometry. We assume that the $\varepsilon$ corresponding to the $\Omega_{\varepsilon}$, which approximates $\Omega$, is given by

$$
\varepsilon=\frac{1}{\left\lceil\sqrt{\frac{n_{r}}{N}}\right\rceil},
$$

with $\lceil\cdot\rfloor$ representing the nearest integer function. As we see in (14), $\varepsilon$ decreases as $N$ decreases. This leads to a subtlety in choosing $N$. We would like $Y^{*}$ to capture the random nature of $\Omega$ while still containing a sufficiently small number $N$ of pores to ensure $\varepsilon$ is small. In the simulations presented here, we take $N=2$ and use (14) above to calculate $u^{\varepsilon}$.

5. Results. In the previous section we developed (8) (with solution $u^{\text {rand }}$ ) to model the flash-heat experiment in porous material and introduced (9) (with solution $u^{\varepsilon}$ ) to approximate (8). Finally, we introduced homogenization theory to approximate (9) with (11) (with solution $U$ ). In this section we will present graphical results corresponding to the three systems and summarize the results of 200 simulations of the three systems. Here, we will use the parameter values listed in Table 1. In each figure $u^{\text {rand }}$ is the 
TABLE 2. In the first three rows, the last three columns contain the average time to calculate the respective solution for the 200 simulations. The last three rows contain the average mesh size over 200 simulations.

\begin{tabular}{|l||l|r|r|r|}
\hline & Model & $\theta=0.9$ & $\theta=0.95$ & $\theta=0.98$ \\
\hline \hline Mean time & $u^{\text {rand }}$ & $45 \mathrm{sec}$ & $53 \mathrm{sec}$ & $62 \mathrm{sec}$ \\
\hline & $u^{\varepsilon}$ & $22 \mathrm{sec}$ & $28 \mathrm{sec}$ & $37 \mathrm{sec}$ \\
\hline & $U$ & $3.9 \mathrm{sec}$ & $4.0 \mathrm{sec}$ & $3.7 \mathrm{sec}$ \\
\hline \hline Mean mesh size & $u^{\text {rand }}$ & 7851 & 8551 & 9965 \\
\hline & $u^{\varepsilon}$ & 5016 & 5744 & 7317 \\
\hline & $U$ & 355 & 355 & 355 \\
\hline
\end{tabular}

solution of (8), $u^{\varepsilon}$ is the solution of (9) and $U$ is the solution of (11). We will consider three different porosity levels, $10 \%$ (or $\theta=0.9$, depicted in Figure 44), $5 \%$ (or $\theta=0.95$, depicted in Figure 5), and 2\% (or $\theta=0.98$, depicted in Figure 6). The results presented here are for $\lambda=10^{-5}$ though we carried out calculations for $\lambda=0$ (the perfectly insulated model) and $\lambda=6 \times 10^{-3}$; each yielded similar results. The random geometries used for the examples are featured in Figures 46 (a), which correspond to $\Omega$ used in (8). The approximations $\Omega_{\varepsilon}$ of $\Omega$ are presented for each level of porosity in Figures 4 6(b). Figures 46 (c) contain plots of the solutions $u^{\text {rand }}, u^{\varepsilon}$ and $U$ at $y=0, t=0.1$ over all values of $x$ on $\omega_{4}$ (the source boundary). In this case, we see that $U$ seems to capture the 'average' behavior of $u^{\text {rand }}$ and that of $u^{\varepsilon}$. We observe that $U$ overestimates when there are large pores near $\omega_{4}$ and underestimates when there are not large pores near $\omega_{4}$. In Figure 4 with $\theta=0.9$ we see that the cluster of pores near $\omega_{4}$ in Figure 4 (a) corresponds to $U$ underestimating $u^{\text {rand }}$ for $x$ greater than 1.25 in Figure 4(c). There are pores near $\omega_{4}$ in Figure 4(b) which correspond to periodic peaks of $u^{\varepsilon}$ above $U$ in Figure 4 (c). There are pores near $\omega_{4}$ in $\Omega_{\varepsilon}$ in Figure 5(b), but they are very small and $U$ overestimates $u^{\varepsilon}$ in Figure 5(c). As should be expected for these systems, the difference between the three solutions seems to decrease with porosity. In Figure 6(c) it is apparent that $U$ is a better estimate for $u^{\text {rand }}$ and $u^{\varepsilon}$ at $\theta=0.98$ than it was for $\theta=0.95$ depicted in Figure 5(c) and $\theta=0.9$ depicted in Figure 4 . Thus we see that $U$ is a better approximation at lower levels of porosity or, equivalently, higher values of $\theta$.

We carried out 200 simulations at each porosity level $(\theta=0.9,0.95$ and 0.98$)$ similar to those used to generate Figures 46 6. For each simulation, we randomly generated the domain $\Omega$ on which we solved (8) for $u^{\mathrm{rand}}$. We also created a random $\Omega_{\varepsilon}$ to solve (9) for $u^{\varepsilon}$. We used the reference cell used to generate $\Omega_{\varepsilon}$ to solve (11) for $U$. In Table 2 we report the average time to solve each of the systems and the average mesh size used to discretize the respective domain. It is clear that of the three systems, (8) is the most computationally intensive. The limit system (11) is the least computationally intensive; its mesh is an order of magnitude smaller than the meshes for the other two systems. Also the computing time required to solve for $U$ is five to ten times less than the time to solve for $u^{\varepsilon}$ and ten to fifteen times less time to solve for $u^{\text {rand }}$. This supports the use of the limit system (11) as a more computationally suitable model than (8) and (9). 


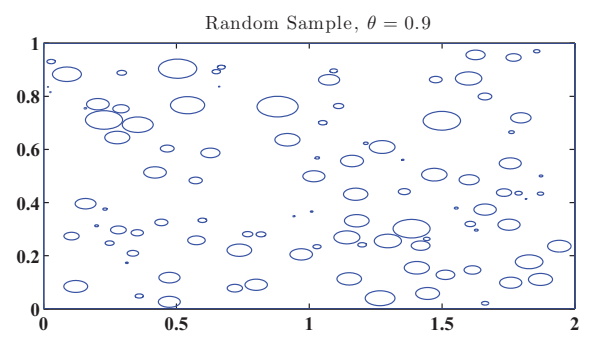

(a)

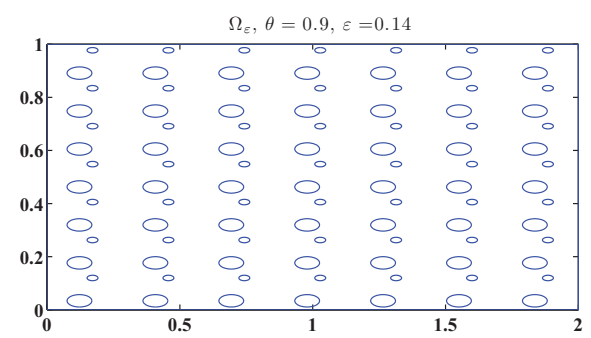

(b)

$$
\varepsilon=0.14, t=0.1 \mathrm{sec}
$$

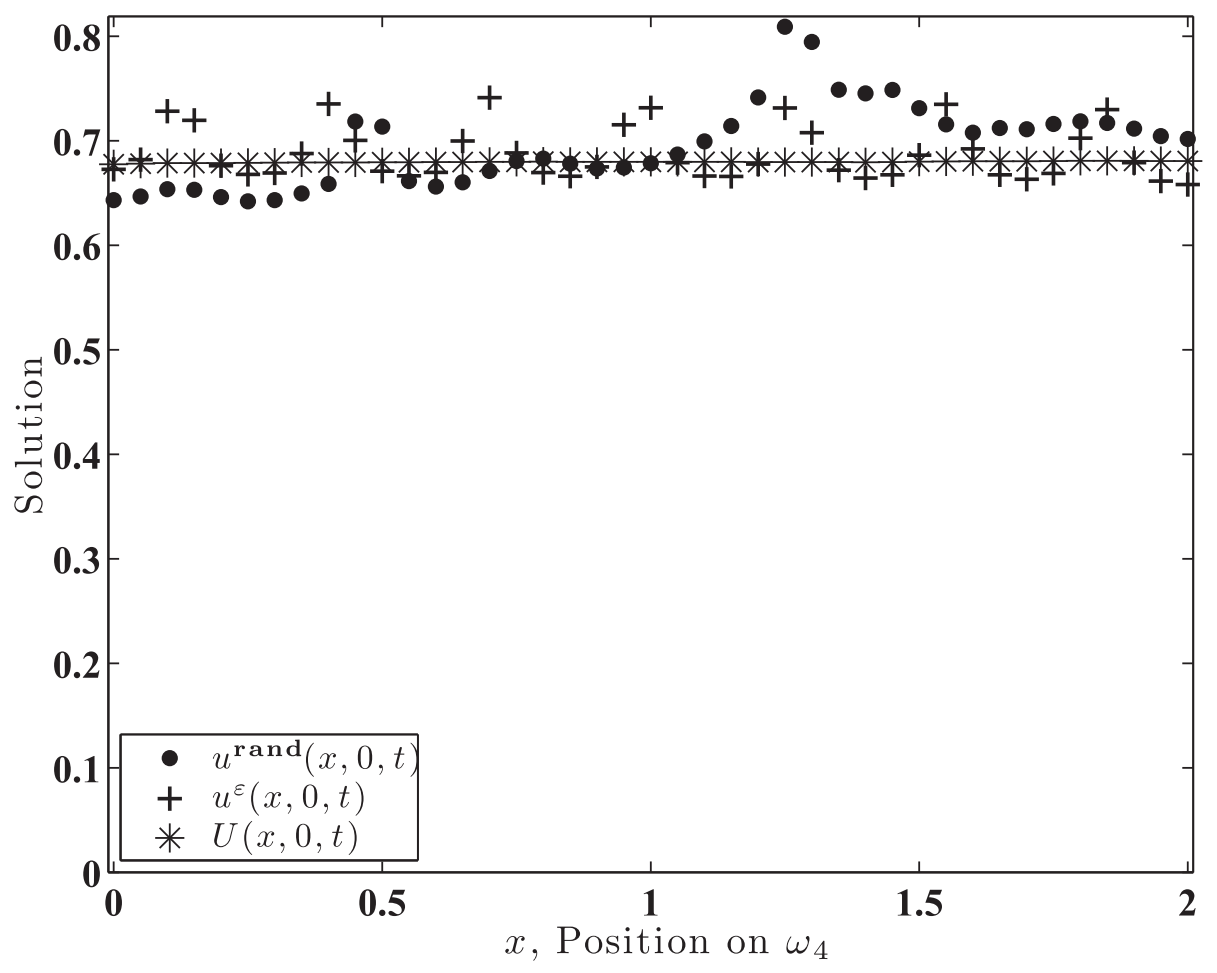

Fig. 4. An example simulation at $\theta=0.9$, or equivalently $10 \%$ porosity. (a) The random geometry $\Omega$; (b) The geometric approximation $\Omega_{\varepsilon}$ of $\Omega$ assumed in homogenization theory, where $\varepsilon=0.14$; (c) $u^{\text {rand }}(x, 0, t), u^{\varepsilon}(x, 0, t)$, and $U(x, 0, t)$ for $t=0.1 \mathrm{sec}$ on $\omega_{4}$. For a representation of the behavior of the three systems over time on the source boundary $\left(\omega_{4}\right)$, see the corresponding movie at http://www4.ncsu.edu/ ${ }^{\sim}$ akcriner/movies/wholerobin9front.zip

The limit system (11) appears to be a good approximation of (8) and (9), at least on the intended observation boundary $\omega_{4}$ in our interrogation experiments. We consider the Frobenius norm of the difference between the limit systems on the source boundary $\omega_{4}$. We will take values at time points $t_{j}=\frac{j-1}{20}$ for $j \in\{1,2, \ldots, 101\}$ and nodes $x_{i}=\frac{i-1}{20}$ for 


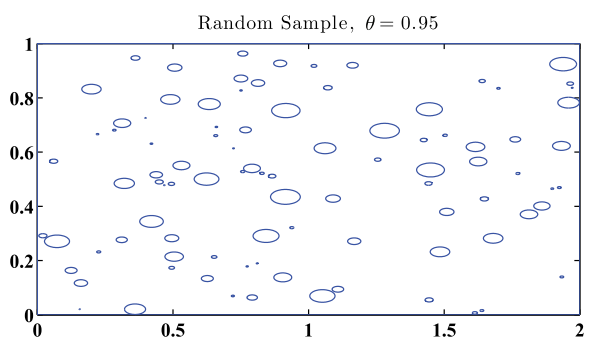

(a)

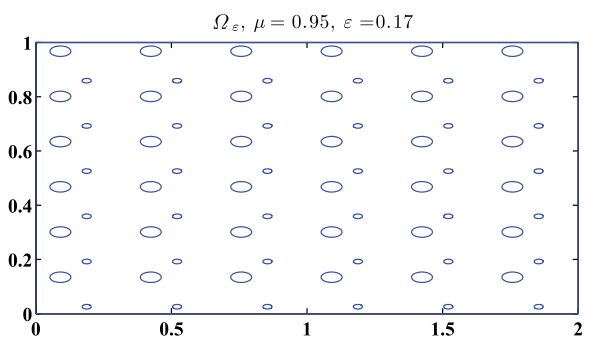

(b)

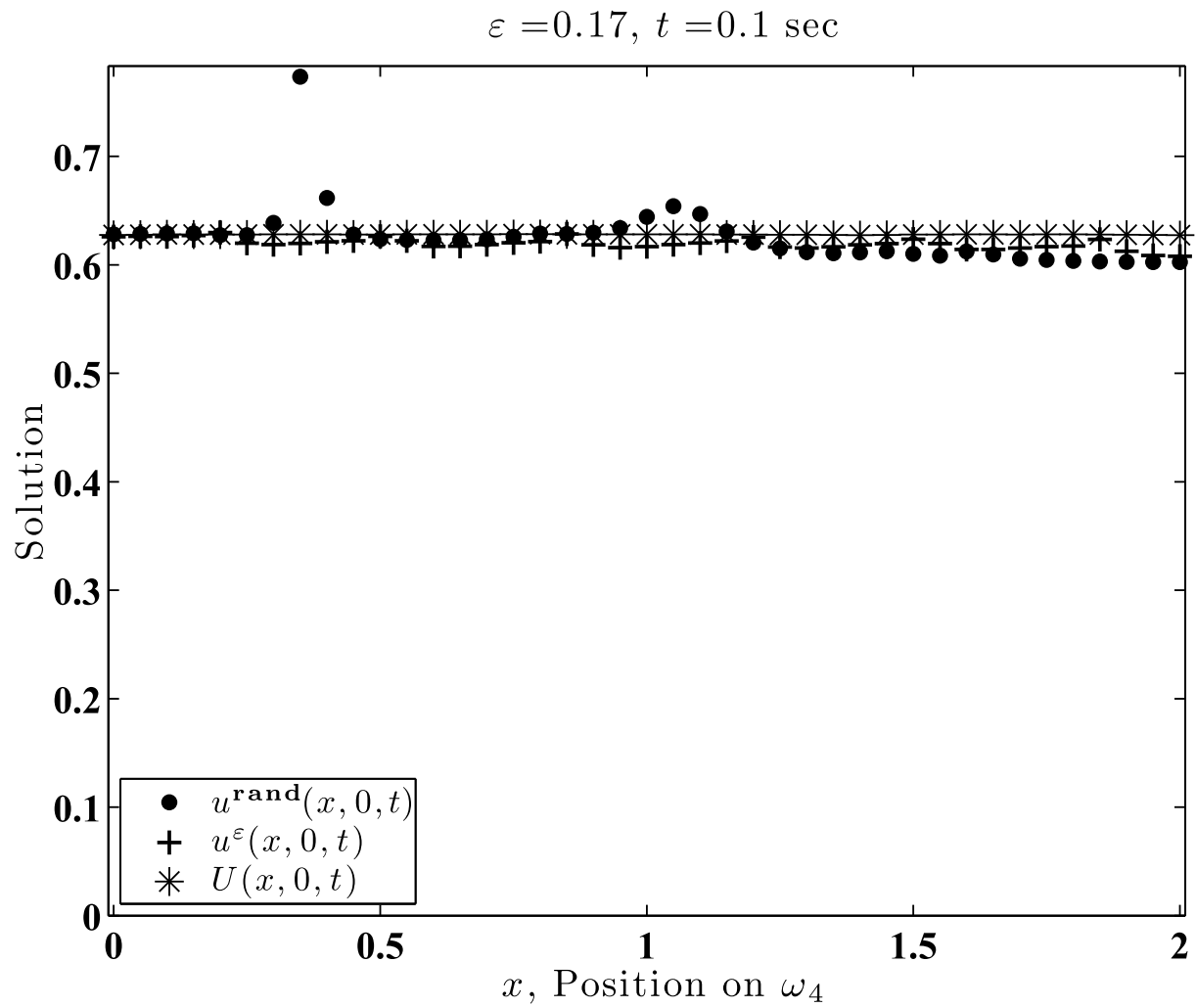

FIG. 5. An example simulation at $\theta=0.95$, or equivalently $5 \%$ porosity. (a) The random geometry $\Omega$; (b) The geometric approximation $\Omega_{\varepsilon}$ of $\Omega$ assumed in homogenization theory, where $\varepsilon=0.17$; (c) $u^{\text {rand }}(x, 0, t), u^{\varepsilon}(x, 0, t)$, and $U(x, 0, t)$ for $t=0.1 \mathrm{sec}$ on $\omega_{4}$. For a representation of the behavior of the three systems over time on the source boundary $\left(\omega_{4}\right)$, see the corresponding movie at http://www4.ncsu.edu/ akcriner/movies/wholerobin95front.zip

$i \in\{1,2, \ldots, 41\}$. For each simulation, we calculated the Frobenius norm of $\left\|U-u^{\text {rand }}\right\|_{F}$,

$$
\left\|U-u^{\mathrm{rand}}\right\|_{F}=\sqrt{\sum_{i=1}^{41} \sum_{j=1}^{101}\left(U\left(x_{i}, 0, t_{j}\right)-u^{\mathrm{rand}}\left(x_{i}, 0, t_{j}\right)\right)^{2}},
$$




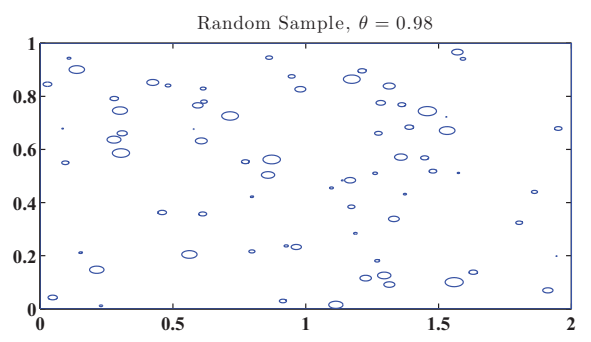

(a)

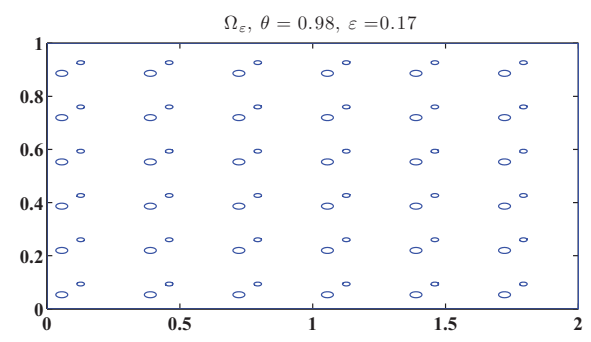

(b)

$\varepsilon=0.17, t=0.1 \mathrm{sec}$

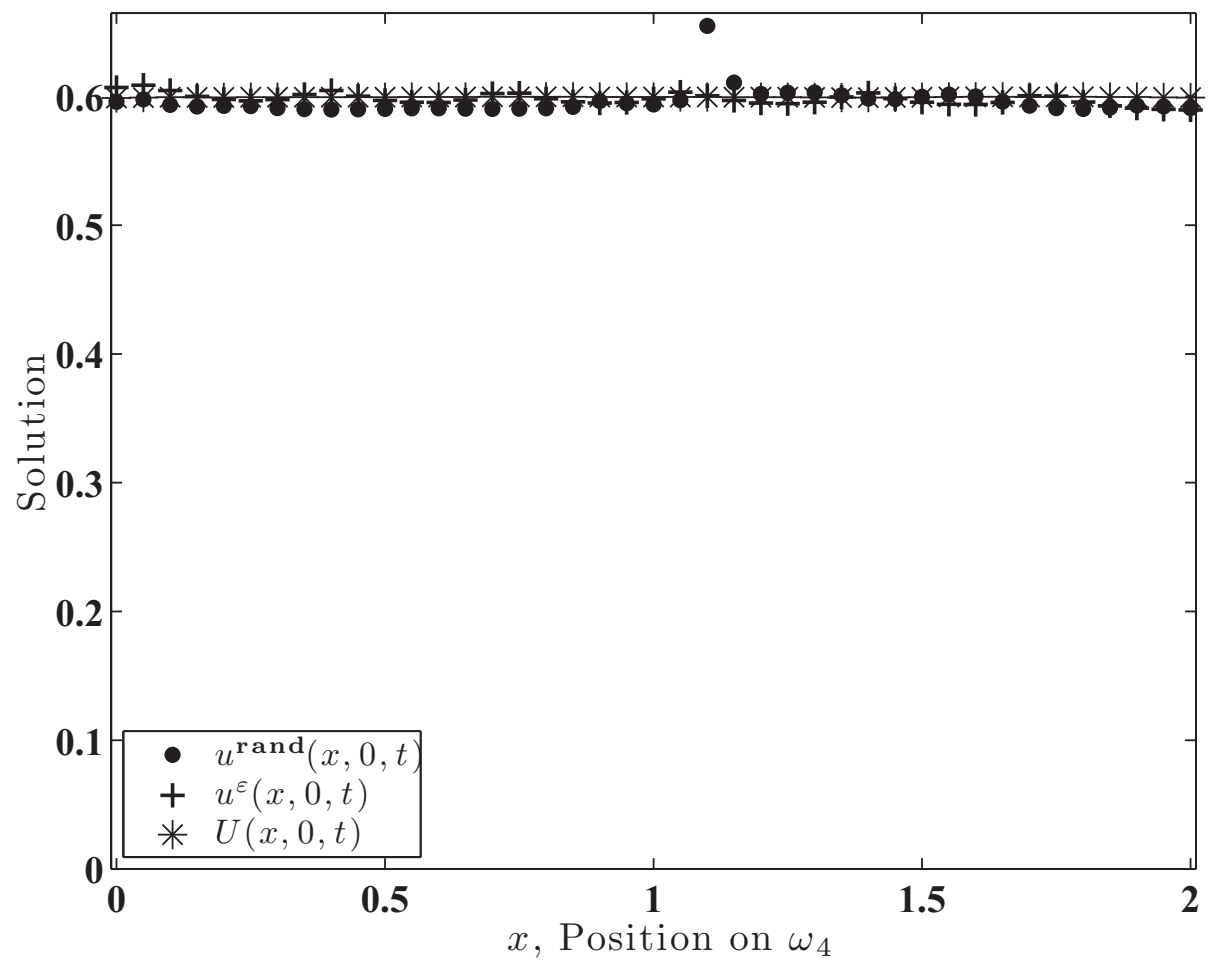

FIG. 6. An example simulation at $\theta=0.98$, or equivalently $2 \%$ porosity. (a) The random geometry $\Omega$; (b) The geometric approximation $\Omega_{\varepsilon}$ of $\Omega$ assumed in homogenization theory, where $\varepsilon=0.17$; (c) $u^{\text {rand }}(x, 0, t), u^{\varepsilon}(x, 0, t)$, and $U(x, 0, t)$ for $t=0.1$ sec on $\omega_{4}$. For a representation of the behavior of the three systems over time on the source boundary $\left(\omega_{4}\right)$, see the corresponding movie at http://www4.ncsu.edu/ ${ }^{\sim}$ akcriner/movies/wholerobin98front.zip

and, similarly, $\left\|U-u^{\varepsilon}\right\|_{F}$. We report the average and standard deviation of these quantities for each porosity level in Table 3. As $\theta$ increases, $U$ better approximates both $u^{\text {rand }}$ and $u^{\varepsilon}$. However, it is the good approximation of $U$ to $u^{\text {rand }}$ which is of primary interest to us since this latter model solution is our intended approximation to the physical problem being investigated. 
TABLE 3. The first and third row contain the mean Frobenius norm of the difference of the solutions for the 200 simulations. The second and fourth row contain the standard deviation of the Frobenius norm of the difference of the solutions for the 200 simulations.

\begin{tabular}{|l||l|l|l|}
\hline & $\theta=0.9$ & $\theta=0.95$ & $\theta=0.98$ \\
\hline \hline Mean $\left\|u^{\text {rand }}-U\right\|_{F}$ & 1.21 & 0.631 & 0.267 \\
\hline St. Dev. $\left\|u^{\text {rand }}-U\right\|_{F}$ & 0.245 & 0.109 & 0.042 \\
\hline Mean $\left\|u^{\varepsilon}-U\right\|_{F}$ & 1.322 & 0.656 & 0.267 \\
\hline St. Dev. $\left\|u^{\varepsilon}-U\right\|_{F}$ & 0.258 & 0.109 & 0.042 \\
\hline
\end{tabular}

6. Conclusion and future work. We have selected and analyzed (3), which mathematically models the flash-heat experiment with imperfect insulation leading to small temperature proportional loss on all of the boundary of the rectangular cross section. The results of homogenization theory in Section 5 are most encouraging. It is also clear from the findings in Section 5 that the choice of reference domain, specifically the location of pores in $\Omega_{\varepsilon}$ in relation to the source boundary $\omega_{4}$, determines the efficacy of $U$ to approximate $u^{\varepsilon}$. Similarly, the location of the pores in $\Omega$ in the random domain determines the behavior of $u^{\text {rand }}$ in relation to the approximation $U$. We also observed that $U$ approximates $u^{\text {rand }}$ best at the lowest level of porosity, $98 \%$ or $\theta=0.98$. We are currently using the limit system (11) developed in Section 4 as a model to carry out parameter estimation on data simulated using (8) with added noise to evaluate the use of the homogenization model to describe noisy experimental data and eventually for use in algorithms to characterize internal damage.

Acknowledgments. This research was supported in part by the National Science Foundation under Research Training Grant (RTG) DMS-0636590 and in part by the Air Force Office of Scientific Research under grant number FA9550-09-1-0226.

\section{REFERENCES}

[1] H.T. Banks, B. Boudreaux, A. K. Criner, K. Foster, C. Uttal, T. Vogel, A.K. Criner and W.P. Winfree, Thermal based damage detection in porous materials, Tech. Rep. CRSC-TR08-11, Center for Research in Scientific Computation, North Carolina State University, Raleigh, NC, September, 2008; Inverse Probl. Sci. Engr., 18 (2009), 835-851.

[2] H.T. Banks, D. Cioranescu, A.K. Criner and W.P. Winfree, Modeling the flash-heat experiment on porous domains, Tech. Rep. CRSC-TR10-06, Center for Research in Scientific Computation, North Carolina State University, Raleigh, NC, May, 2010.

[3] H. T. Banks, N. L. Gibson and W. P. Winfree, Void detection in complex geometries, Tech. Rep. CRSC-TR08-09, Center for Research in Scientific Computation, North Carolina State University, Raleigh, NC, May, 2008.

[4] H. T. Banks, M. L. Joyner, B. Wincheski and W. P. Winfree, Nondestructive evaluation using a reduced-order computational methodology, Inverse Probl. 16(4):929-945, 2000. MR1776475 (2001e:78022)

[5] H.T. Banks and F. Kojima, Boundary shape identification problems in two-dimensional domains related to thermal testing of materials, Quart. Appl. Math. 47 (2):273-293, 1989. MR0998101 (90f:65168)

[6] H. T. Banks and F. Kojima, Identification of material damage in two-dimensional domains using the SQUID-based nondestructive evaluation system, Inverse Probl. 18 (6):1831-1855, 2002. MR1955921 
[7] H. T. Banks, F. Kojima and W. P. Winfree, Boundary estimation problems arising in thermal tomography, Inverse Probl. 6 (6):897-921, 1990. MR.1082231 (91k:80003)

[8] D. Cioranescu, A. Damlamian and G. Griso, The periodic unfolding method in domains with holes, to appear.

[9] D. Cioranescu and J. Saint Jean Paulin. Homogenization of Reticulated Structures, Volume 136 of Applied Mathematical Sciences, Springer-Verlag, New York, 1999. MR1676922 (2000d:74064)

[10] D. Cioranescu and P. Donato, An Introduction to Homogenization, Oxford Lecture Series in Mathematics and Its Applications Volume 27, Oxford University Press, New York, 1999. MR1765047 (2001j:35019)

[11] P. Donato and A. Nabil, Homogenization and correctors for the heat equation in perforated domains, Ric. Mat., 50(1):115-144, 2001. MR1941824(2003i:35017)

[12] The Mathworks, Inc., Partial Differential Equation Toolbox 1: User's Guide, The Mathworks, Inc., Natick, MA, 2008.

[13] W. J. Parker, R. J. Jenkins, C. P. Butler and G. L. Abbott, Flash method of determining thermal diffusivity, heat capacity, and thermal conductivity, J. Appl. Phys. 32 (9):1679-1684, 1961.

[14] Pavel S̆olin, Partial Differential Equations and the Finite Element Method, John Wiley \& Sons, Inc., Hoboken, NJ, 2006. MR2180081 (2006f:35004)

[15] Wenping Wang, Jiaye Wang and Myung-Soo Kim, An algebraic condition for the separation of two ellipsoids, Comput. Aided Geom. Design, 18(6):531-539, 2001. MR1843064 (2002c:65030) 